\title{
Development of Extended Two-Point Model for Asymmetric Scrape-Off Layer*)
}

\author{
Apiwat WISITSORASAK ${ }^{1,2)}$, Sébastien KAHN ${ }^{3)}$, Bernard PÉGOURIÉ ${ }^{3)}$, \\ Jean-Francois ARTAUD ${ }^{3)}$, Guido CIRAOLO ${ }^{3)}$ and Cédric REUX ${ }^{3)}$ \\ ${ }^{1)}$ Department of Physics, King Mongkut's University of Technology Thonburi, Bangkok, Thailand \\ ${ }^{2}$ Theoretical and Computational Science Center, King Mongkut's University of \\ Technology Thonburi, Bangkok, Thailand \\ ${ }^{3)}$ CEA, IRFM, F-13108 Saint-Paul-les-Durance, France
}

(Received 8 January 2019 / Accepted 22 July 2019)

\begin{abstract}
The SYCOMORE code is a modular system code which aims at modelling future fusion power plants with all subsystems and to provide a global view of the whole plant. The code consists in different modules handling the different subsystems of the plant, from the core plasma to the conversion of heat to electricity. Among them, the divertor is one of the most important components and must withstand high heat load. While the complex magnetic configuration in tokamaks and the peculiar transport in the scrape-off layer (SOL) give rise to an asymmetry in the high field and low field energy fluxes, this issue should be properly addressed in SYCOMORE for quick and reliable predictions. In this work, the SOLDIV code which is a scrape-off-layer and divertor module in SYCOMORE has been used to investigate this asymmetry problem based on an extended two-point model. When the outgoing fluxes of particles and heat from the plasma core enter the SOL at the stagnation point, they split into two parts: one transporting to the inner divertor, and the other transporting to the outer divertor. By introducing the imbalance factor of the energy flux between the two divertor plates, the transport equations become a set of nonlinear equations that can be numerically solved for the densities and temperatures at both divertor plates and the stagnation point. Strong temperature and density differences at the targets can be found. The analysis results are validated with the transport code SolEdge2D-EIRENE for WEST test discharges. The simulation results for ITER are also investigated.
\end{abstract}

(c) 2019 The Japan Society of Plasma Science and Nuclear Fusion Research

Keywords: divertor, SOL-divertor, system code, two-point model, balloon transport

DOI: $10.1585 /$ pfr. 14.3403150

\section{Introduction}

Fusion energy is a highly promising potential candidate that offers an almost inexhaustible source of energy for future generations, and low environmental pollution. However, one of the greatest challenges in the success of the magnetic confinement fusion devices, such as ITER and DEMOs, may be related to the turbulent dynamics of the plasma in the scrape-off layer (SOL), the narrow region surrounding the core plasma where the magnetic field lines are open and terminate on the vessel of the device [1-3]. The plasma behaviour in this region not only governs the overall confinement properties of the device, but also regulates the impurity dynamics which can dilute the fusion fuel and radiate a part of the plasma energy content [4]. The SOL plasma also determines the conducted/convected power exhausted at the reactor vessel walls [5]. This issue is critically important since a too high heat load can severely damage the plasma-facing material

author'se-mail: apiwat.wis@kmutt.ac.th

*) This article is based on the presentation at the 27th International Toki Conference (ITC27) \& the 13th Asia Pacific Plasma Theory Conference (APPTC2018). which can make fusion reactors not economically attractive or even feasible. While diagnostic access for the SOL plasma is difficult and is limited, simulation of the plasma dynamics in the SOL region has proven to be an invaluable tool to address the physics of this region.

The simulation of a whole tokamak is a complex problem because it involves different phenomena and all subsystems are not entirely independent from each other. In order to understand and design a whole fusion plant, a fast and reliable simulation code is inevitable [6,7]. The SYCOMORE code is a modular system code which aims at modelling future fusion power plants with all subsystems and to provide a global view of the whole plant $[8,9]$. The code consists in different modules handling the different subsystems of the plant, from the core plasma to the conversion of heat to electricity [10]. The divertor is one of the most important components of the reactor and may be exposed to excessive heat and particle fluxes. While the complex magnetic configuration in tokamaks and the peculiar transport in the SOL give rise to the asymmetry in the high field and low field energy fluxes [11, 12], 
this issue should be properly addressed in SYCOMORE for quick and reliable predictions. In this work, the SOLDIV code which is a scrape-off-layer and divertor module in SYCOMORE has been used to investigate this asymmetry problem based on an extended two-point model. When the outgoing fluxes of particles and heat from the plasma core enter the SOL at the stagnation point, they split into two parts: one transporting to the inner divertor, and the other transporting to the outer divertor. By introducing the imbalance factor of the energy flux, the transport equations become a set of nonlinear equations that can be numerically solved for the densities and temperatures at both divertor plates and the stagnation point. Strong temperature and density differences at the targets can be found. The analysis results are then validated with the transport code SolEdge2D-EIRENE for WEST discharges. The simulation results for ITER are also investigated.

This paper is organised as follows. We first describe the extended two-point model and the algorithm for solving the model in section 2. Section 3 shows the comparison with the SolEdge2D-EIRENE code and simulation predictions for ITER. This work is summarised in section 4.

\section{Methodology}

The basic two-point model describes the parallel transport along a magnetic field line [2]. The field line can simply be straightened to describe the transport in the parallel direction. This parallel transport is assumed to be decoupled from the perpendicular one. The most important feature of the two-point model is to relate the physical quantities at two points: 1) the upstream point where the heat flux and particles enter the SOL, and 2) the divertor surface where the particles strike the solid material. In this model, the sources and sinks are treated locally at the boundaries. The locations of these two points are illustrated in Fig. 1.

The two-point model can be directly derived from the fluid equations for plasma in steady state condition [2]. The particle and heat fluxes leave the core and enter the SOL at the upstream point. It is further assumed that neutrals recycling from the targets are ionized in a thin layer in front of the target. For each side of the SOL flux tube, if it is assumed that the fraction of the momentum loss in the plasma flow is $f_{\text {mom }}[1]$, the total pressure throughout the entire length of the flux tube can be written as:

$$
f_{\text {mom }} n_{u} T_{u}\left(1+M_{u}^{2}\right)=n_{t} T_{t}\left(1+M_{t}^{2}\right),
$$

where $M$ is the Mach number, and the subscribes $u$ and $t$ refer to the values at the upstream and target. Here we also assume that the ion and electron temperatures are equal, $T_{i}=T_{e}$. Note that the Mach number at the target, $M_{t}$, is approximately equal to unity. Equation 1 then becomes:

$$
f_{\text {mom }} n_{u} T_{u}\left(1+M_{u}^{2}\right) \approx 2 n_{t} T_{t}
$$

The total particle flux at the upstream point may be large during pellet-fuelling operation, and it may change the up-

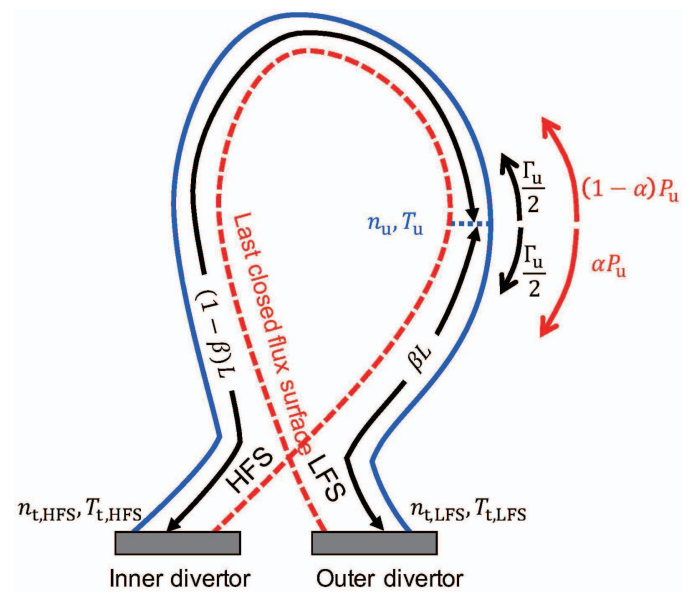

Fig. 1 The particle flux, $\Gamma_{u}$, from the core enters the SOL and equally separates into two parts. On contrary, the heat flux splits asymmetrically. The power to the outer and inner divertors are $\alpha P_{u}$ and $(1-\alpha) P_{u}$, respectively. The connection length of the outer leg is $\beta L$ and the connection length of the inner leg is $(1-\beta) L$.

stream boundary significantly. One can define the parallel flux entering the SOL as $G=\left(q_{95} \Gamma_{u}\right) /(2 \pi a \kappa \lambda)$, where $q_{95}$ is the safety factor at $95 \%$ of the poloidal flux, $\Gamma_{u}$ the total rate of particle leaving the confined plasma volume, $a$ the minor radius, $\kappa$ the elongation, and $\lambda$ the characteristic SOL width [13]. The parallel particle flux can be also written as $G=n M c_{s}$, where $c_{s}$ is the sound speed. In the SOL, the pressure conservation requires that $n T\left(1+M^{2}\right)$ is constant along the field lines. In the heat transport equation, the parallel heat conduction generally dominates over the parallel heat convection [2]. Therefore the heat flux does not depend on the density and Mach number. Therefore the temperature remains unchanged, i.e. $T=T_{u}$. The pressure conservation at the upstream in the presence and absence of the particle fluxes can then be written as

$$
n T_{u}\left(1+M_{u}^{2}\right)=n_{u} T_{u}
$$

Combining this equation with the expression of $G$, one obtains

$$
G\left(1+M_{u}^{2}\right)=n_{u} M_{u} c_{s} .
$$

Solving for $M_{u}$ by using the quadratic formula, the Mach number at the upstream depends on the parallel particle flux as

$$
M_{u}=\frac{1}{2}\left(n_{u} c_{s} / G-\sqrt{\left(n_{u} c_{s} / G\right)^{2}-4}\right) .
$$

After integrating along the field line of the flux tube, we finally obtain the heat transport equation [2]:

$$
T_{u}^{7 / 2}=T_{t}^{7 / 2}+\frac{7 f_{\text {cond }} q_{\|} L}{2 \kappa_{0 e}},
$$

where $f_{\text {cond }}$ denotes the fraction of the ratio of the heat flux transported by conduction to the total heat flux, $\kappa_{0 e}$ is the electron parallel conductivity, and $q_{\|}$is the parallel heat 
flux. Note that, in this model, no volumertric particle or power sources or sinks are considered. The ionization and recycling of neutrals are assumed to occurr only in a thin region near the target. Thus we write

$$
\left(1-f_{\text {power }}\right) q_{\|, L F S}=e \gamma \Gamma_{t} T_{t, L F S}\left(1+\frac{\epsilon}{\gamma T_{t, L F S}}\right),
$$

where $f_{\text {power }}$ is the fraction of the parallel heat flux that is lost before reaching the target, $\epsilon$ is the average energy lost by the electrons per recycled atom and approximately equals $25 \mathrm{eV}$ [8], and $\gamma$ is the sheath heat transmission coefficient, $\gamma \approx 7$ [2].

The asymmetries of the heat fluxe in the SOL can be modelled by introducing imbalance factors. The particle and heat fluxes enter the SOL at the upstream point, see Fig. 1. The precise location of the upstream is determined by the parameter $\beta$ which is the fraction of the LFS connection length to the total length. The particle flux $\left(\Gamma_{u}\right)$ from the core to the SOL then splits equally into two parts because $n_{\mathrm{u}, \text { left }}=n_{\mathrm{u}, \text { right }}, T_{\mathrm{u}, \text { left }}=T_{\mathrm{u}, \text { right }}$, and the net Mach number equals zero at the stagnation point. One part of the fluxes goes to the outer target and the other directs towards the inner target.

For the heat flux from the core to the SOL, it enters the SOL at the upstream point as the particle flux does. We assume that it then splits asymmetrically into two parts. The amount of the power to the outer divertor is $\alpha P_{u}$, and the remaining power $(1-\alpha) P_{u}$ directs towards the inner target. The imbalance factors, $\alpha$ and $\beta$, are inserted in the calculation of the two-point model leading to different sets of equations for the low-field side (LFS) and high-field side (HFS) as follows

for LFS:

$$
\begin{aligned}
& 2 n_{t, L F S} T_{t, L F S}=n_{u} T_{u, L F S} \times\left(1+M_{u, L F S}^{2}\right) f_{\mathrm{mom}}, \\
& T_{u, L F S}^{7 / 2}=T_{t, L F S}^{7 / 2}+\frac{7 f_{\mathrm{cond}} \alpha q_{\|} \beta L}{2 \kappa_{0 e}}, \\
& \left(1-f_{\text {power }}\right) \alpha q_{\|}=e \gamma \Gamma_{t} T_{t, L F S} \times\left(1+\frac{\epsilon}{\gamma T_{t, L F S}}\right),
\end{aligned}
$$

for HFS:

$$
\begin{gathered}
2 n_{t, H F S} T_{t, H F S}=n_{u} T_{u, H F S} \times\left(1+M_{u, H F S}^{2}\right) f_{\mathrm{mom}}, \\
\begin{aligned}
& T_{u, H F S}^{7 / 2}=T_{t, H F S}^{7 / 2}+\frac{7 f_{\text {cond }}(1-\alpha) q_{\|}(1-\beta) L}{2 \kappa_{0 e}}, \\
&\left(1-f_{\text {power }}\right)(1-\alpha) q_{\|}= e \gamma \Gamma_{t} T_{t, H F S} \\
& \times\left(1+\frac{\epsilon}{\gamma T_{t, H F S}}\right) .
\end{aligned}
\end{gathered}
$$

At the upstream point, the densities and temperatures of the two field sides are equal. Therefore we have additional constrains at the upstream point.

$$
\begin{aligned}
& n_{u, H F S}=n_{u, L F S}=n_{u}, \\
& T_{u, H F S}=T_{u, L F S}=T_{u} .
\end{aligned}
$$

In principle, the imbalance factors, $\alpha$ and $\beta$, can be calculated from kinetic theory.

The extended two-point model consists in a set of six nonlinear equations, equations $8-13$, with 8 unknowns: $n_{t, L F S}, n_{t, H F S}, T_{t, L F S}, T_{t, H F S}, T_{u}, n_{u}, \alpha$ and $\beta$. Since $q_{\|}$is given from the core module HELIOS [14] and $n_{u}$ is the main control parameter, an additional parameter must be fixed for the system to be solved. It was chosen $\alpha$ in this work. For each set of equations, one can derive an expression that only depends on the target temperature, $T_{t}$, as follows

$$
\begin{aligned}
T_{t, L F S}= & \left(1+\frac{\epsilon}{\gamma T_{t, L F S}}\right)^{-2}\left(\gamma\left(1+M_{L F S}^{2}\right) f_{\mathrm{mom}} n_{u}\right)^{-2} \\
& \times \frac{2 m_{i}\left(\alpha q_{\|}\right)^{2}\left(1-f_{\mathrm{power}}\right)^{2}}{\left(T_{t, L F S}^{7 / 2}+\frac{7 f_{\mathrm{cond}} \alpha q_{\|} \beta L}{2 \kappa_{0 e}}\right)^{4 / 7}} \\
T_{t, H F S}= & \left(1+\frac{\epsilon}{\gamma T_{t, H F S}}\right)^{-2}\left(\gamma\left(1+M_{H F S}^{2}\right) f_{\mathrm{mom}} n_{u}\right)^{-2} \\
& \times \frac{2 m_{i}\left((1-\alpha) q_{\|}\right)^{2}\left(1-f_{\mathrm{power}}\right)^{2}}{\left(T_{t, H F S}^{7 / 2}+\frac{7 f_{\mathrm{cond}}(1-\alpha) q_{\|}(1-\beta) L}{2 \kappa_{0 e}}\right)^{4 / 7}}
\end{aligned}
$$

Note that $f_{\text {mom }}$ is a function of the target temperature [1]. In this work, the conduction factor $f_{\text {cond }}$ is kept constant and approximately equals one. The target temperature $\left(T_{t}\right)$ of each side can be independently determined as a function of $\alpha$ or $\beta$ using the bisection method [15]. Once the target temperatures at both targets are obtained, they are substituted back into the other equations to directly obtain $n_{t}$ at each side and $T_{u}$. This can be done by the bisection method, see Fig. 2. By equating the upstream temperature from equations 9 and 12, and assuming $T_{t, L F S}>T_{t, H F S}$, one can also obtain an inequality relation between $\alpha$ and $\beta$ :

$$
\frac{\alpha}{1-\alpha} \leq \frac{1-\beta}{\beta} \text {. }
$$

Once the upstream temperature and density are determined, the actual power lost in the SOL can be computed through impurity radiation. The heat balance between the upstream and the target points leads to the expression:

$$
F_{\text {imp }}=\left(\frac{f_{\text {power }} q_{\|}}{2 n_{u} \kappa_{0 e} T_{u}^{2} \int_{0}^{T_{u}} \frac{1}{2} T^{0.5} L_{Z}(T) d T}\right)^{2},
$$

where $F_{\text {imp }}=f_{\text {imp }} / Z_{\text {eff }}$ [8]. After $F_{\text {imp }}$ is determined, the impurity fraction $f_{\text {imp }}$ is then obtained by

$$
f_{\mathrm{imp}}=\frac{F_{\mathrm{imp}}}{1-0.01 F_{\mathrm{imp}} Z_{\mathrm{imp}}\left(Z_{\mathrm{imp}}-1\right)},
$$

where $Z_{\text {imp }}$ is the charge number of the seeded impurity [16], i.e. $Z_{\mathrm{Ar}}=18$ for argon. The integration in equation $19, \int_{0}^{T_{u}} \frac{1}{2} T^{0.5} L_{Z}(T) d T$, is an impurity radiation loss function which can be calculated numerically from the interpolation of experimental data $[16,17]$. It is worth noting that the integral assumes a target temperature equal to zero. This assumption reduces the computational time, but also 
tends to overestimate the radiation losses. The value of $f_{\text {power }}$ corresponding to the impurity fraction can be finally obtained. Then the peak on the inner/outer divertor target $q_{\text {peak,in/out }}$ is

$$
\begin{aligned}
q_{\text {peak }, \text { in } / \text { out }}= & \frac{P_{\text {separatrix }}\left(1-f_{\text {power }}\right)-P_{\text {rad,H }}}{A_{\text {wet,in/out }}} \\
& +\frac{f_{\text {power }} P_{\text {separatrix }}}{A_{\text {wet,in/out }}} f_{\text {div }} .
\end{aligned}
$$

$P_{\mathrm{rad}, \mathrm{H}}=e\left(\epsilon-E_{i}\right) n_{\mathrm{t}} \sqrt{\frac{2 e T_{\mathrm{t}}}{m_{i}}} \frac{4 \pi a \lambda}{q_{95}}$ is an hydrogen radiation power. $E_{i}$ is the ionization energy of hydrogen $(13.6 \mathrm{eV})$. $A_{\text {wet }}$ is the plasma-wetted area of a divertor. $f_{\text {div }}$ is the fraction of radiated power from the SOL region that goes to the divertor walls. Since the peak heat flux on the target plates cannot exceed $q_{\text {threshold }}=10 \mathrm{MW} / \mathrm{m}^{2}$ (generally, limitation occurs at the outer divertor) [18], an impurity such as argon is introduced (parameter $f_{\text {imp }}$ ) for increasing the radiation in the SOL and limiting the heat load on the targets [19].

The work flow of the algorithm for solving the extended two-point model is illustrated in Fig. 2 and is implemented in the new SOLDIV subroutine. This subroutine initially takes inputs including geometric parameters ( $\left.R, a, L, \lambda, A_{\mathrm{wet}}, q_{95}\right)$, the power crossing the separatrix $P_{u}$, the additional parameter $\alpha$ or $\beta$ required for solving the system of equations as explained above, and a first guess for the impurity fraction $f_{\text {imp }}$. The upstream density, $n_{u}$, is

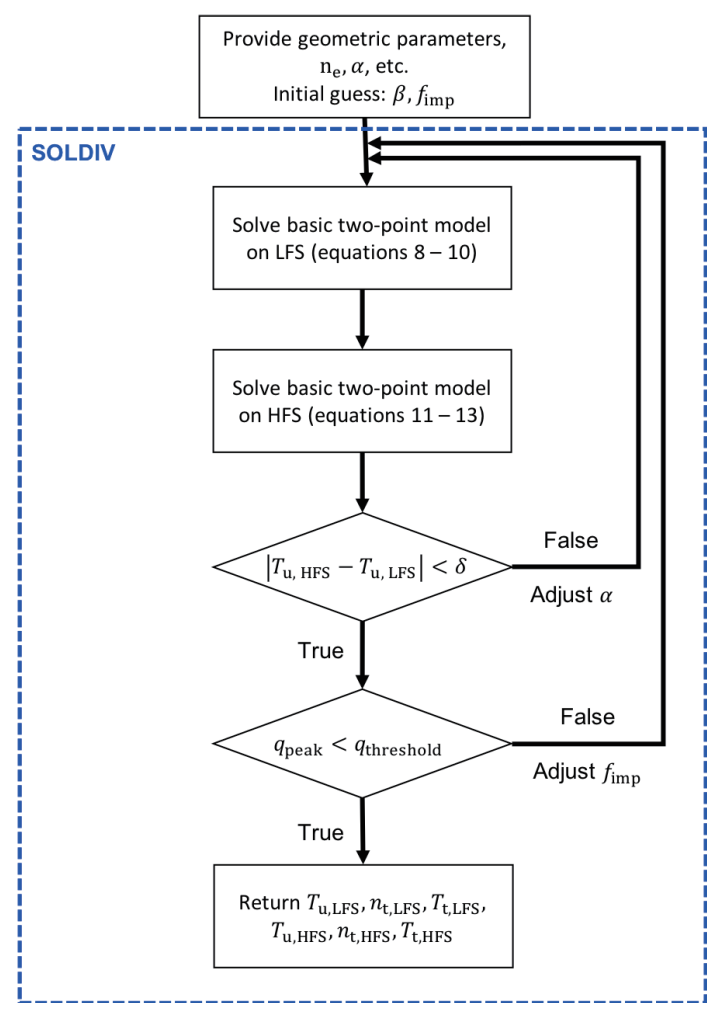

Fig. 2 The flow chart describes the work flow for solving the extended two-point model. Note that $\delta$ denotes the tolerance of the temperature difference and $q_{\text {threshold }}$ represents the threshold value of the heat flux on a divertor target. the control parameter chosen by the operator from physical considerations (generally a fraction of the pedestal density defined in the HELIOS core module).

\section{Result and Discussion \\ 3.1 Comparison with SolEdge2D-EIRENE code simulation}

In this section, we validate the simulation results from the extended two-point model with the transport code SolEdge2D-EIRENE. SolEdge2D is a two-dimensional transport code for simulating the edge plasma with complex and realistic 2D magnetic and wall geometries [20, 21]. Its calculation is based on a fluid model that includes the conservation equations for the particles, for the momentum, and for the energy for both electrons and ions. It also assumes toroidal-axis symmetry and the computational domain is then reduced to a $2 \mathrm{D}$ poloidal crosssection. SolEdge2D can be coupled with EIRENE to investigate edge plasma transport, thermal exhaust and detachment behavior in tokamak devices [22].

In order to compare the results from the two codes, the two-point model takes the upstream density and the power through the separatrix from the SolEdge2D-EIRENE code as input parameters. The two-point model is then used to predict the upstream and target temperatures, and the target densities. Simulation results of two WEST discharges, 53259 and 54034, are chosen for the comparison. Table 1 shows the summary of these two discharges. We note that the discharges labelled 54034A and 54034B are from the same discharge, but with different phases. For the WEST configuration, the plasma major radius is $R=2.5 \mathrm{~m}$, the

Table 1 Summary of SolEdge2D-EIRENE code simulations for WEST test discharges. Note that $P_{\text {sep }}$ is the power through the separatrix [MW], $P_{\text {rad,SOL }}$ is the total power radiated in the SOL [MW], $\lambda_{q}$ is the scale length for the power decay $[\mathrm{mm}]$. The densities are reported in a unit of $10^{19} \mathrm{~m}^{-3}$, and the temperatures are measured in a unit of eV.

\begin{tabular}{cccc}
\hline Parameters & 53259 & $54034 \mathrm{~A}$ & $54034 \mathrm{~B}$ \\
\hline$P_{\text {sep }}$ & 1.0 & 1.8 & 2.0 \\
$P_{\text {rad,SOL }}$ & 0 & 0.50 & 0.10 \\
$\lambda_{q}$ & 8.5 & 8.7 & 8.5 \\
$n_{u}$ & 0.6 & 1.6 & 1.2 \\
$n_{\mathrm{t}, \mathrm{HFS}}$ & 0.28 & 1.3 & 3.5 \\
$n_{\mathrm{t}, \mathrm{LFS}}$ & 0.25 & 1.2 & 2.2 \\
$T_{u}$ & 110 & 100 & 100 \\
$T_{\mathrm{t}, \mathrm{HFS}}$ & 102 & 75 & 50 \\
$T_{\mathrm{t}, \mathrm{LFS}}$ & 103 & 76 & 57 \\
\hline
\end{tabular}



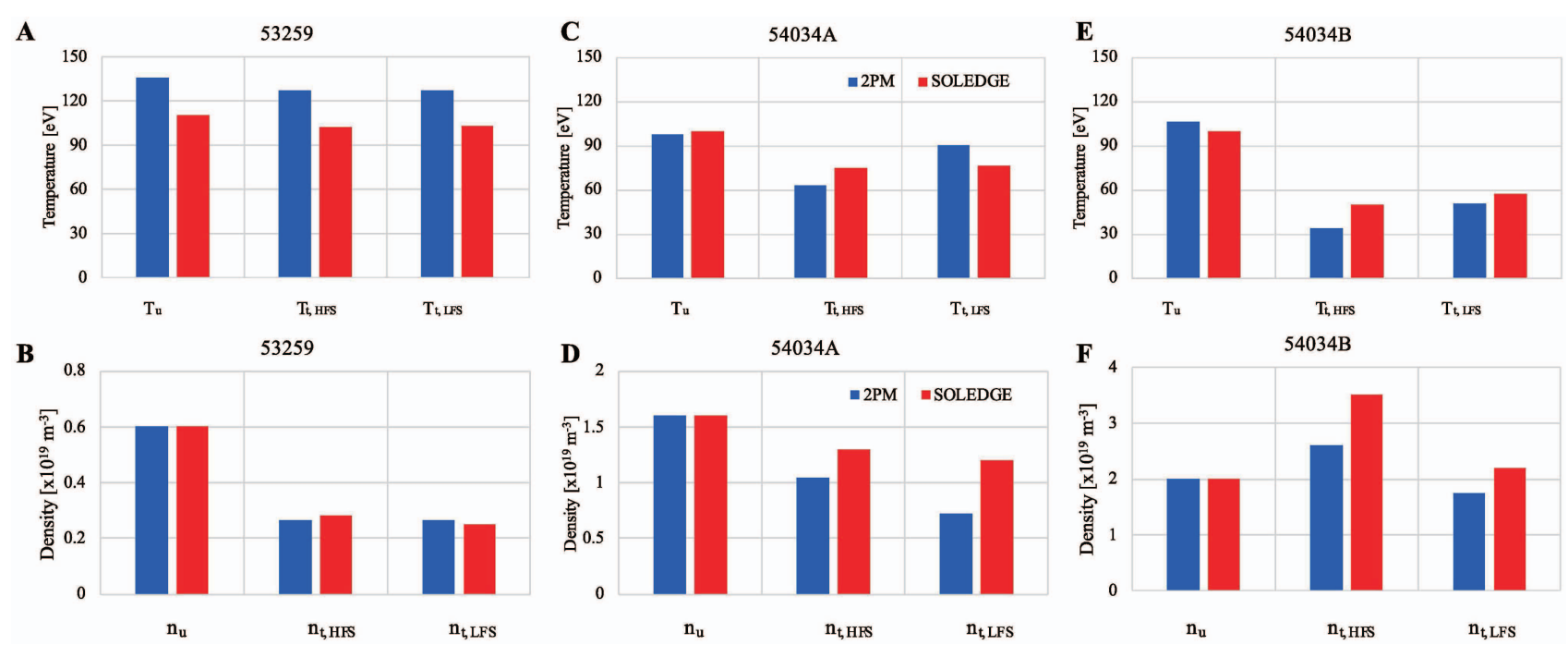

Fig. 3 Comparison of the simulation results between the extended two-point model (2PM) and the transport code SolEdge2D-EIRENE.

minor radius $a=0.5 \mathrm{~m}$, the elongation $\kappa=1.54$, the toroidal magnetic field $B_{\phi}=3.6 \mathrm{~T}$ and the plasma current $I_{P}=0.50 \mathrm{MA}$.

Figure 3 shows the comparison of the simulation results of the densities and temperatures for the two discharges as obtained by the two codes. The temperatures at the upstream $\left(T_{u}\right)$, and the inner $\left(T_{\mathrm{t}, \mathrm{HFS}}\right)$ and outer $\left(T_{\mathrm{t}, \mathrm{LFS}}\right)$ targets are illustrated in the top row, and the densities at the upstream $\left(n_{u}\right)$, and the inner $\left(n_{\mathrm{t}, \mathrm{HFS}}\right)$ and outer $\left(n_{\mathrm{t}, \mathrm{LFS}}\right)$ targets are presented in the bottom row. As we can see in these plots, the calculation from the extended two-point model overall agree with the SolEdge2D-EIRENE simulation.

We also compute the percentage differences between the results from both simulation codes. Here the percentage difference is defined as

$$
\Delta(\%) \equiv \frac{f_{2 \mathrm{PM}}-f_{\text {SolEdge }}}{f_{\text {SolEdge }}} \times 100,
$$

where $f_{2 \mathrm{PM}}$ and $f_{\text {SolEdge }}$ denote the same physical quantities of the plasma (i.e. $n_{\mathrm{t}, \mathrm{HFS}}, n_{\mathrm{t}, \mathrm{LFS}}, T_{u}, T_{\mathrm{t}, \mathrm{HFS}}$, and $T_{\mathrm{t}, \mathrm{LFS}}$ ) which are obtained from the two-point model (2PM) and the SolEdge2D-EIRENE simulation, respectively. We further note that, in this part, the fraction of power to the lowfield side $(\alpha)$ is determined by optimising the total percentage differences of the densities and temperatures at the upstream point and the inner and outer targets. These imbalance factors are presented in Table 3. When $\alpha$ is not equal to 0.50 , the differences of the densities and temperatures at the targets become more obvious. Table 2 shows the percentage differences for these discharges and their average. As a whole, the average percentage difference for the densities and the temperatures at the upstream and the targets are less than $18 \%$.

This work only compares the calculation from the extended two-point model with SolEdge2D-EIRENE simulation on test discharges from WEST. However, it is a
Table 2 The percentage differences of the densities and temperatures of the two discharges from the two-point model and SolEdge2D-EIRENE.

\begin{tabular}{ccccc}
\hline Quantities & 53259 & $54034 \mathrm{~A}$ & $54034 \mathrm{~B}$ & Average \\
\hline$n_{\mathrm{t}, \mathrm{HFS}}$ & -4.98 & -19.74 & -25.29 & -16.67 \\
$n_{\mathrm{t}, \mathrm{LFS}}$ & 6.42 & -39.61 & -20.17 & -17.79 \\
$T_{u}$ & 23.13 & -2.44 & 5.99 & 8.89 \\
$T_{\mathrm{t}, \mathrm{HFS}}$ & 24.58 & -15.90 & -32.61 & -7.98 \\
$T_{\mathrm{t}, \mathrm{LFS}}$ & 23.37 & 19.05 & -10.86 & 10.52 \\
\hline
\end{tabular}

Table 3 The fraction of the power to the LFS $(\alpha)$ and the fraction of the LFS to the total connection length $(\beta)$ as calculated from the extended two-point model.

\begin{tabular}{cccc}
\hline Imbalance factors & 53259 & $54034 \mathrm{~A}$ & $54034 \mathrm{~B}$ \\
\hline$\alpha$ & 0.50 & 0.54 & 0.52 \\
$\beta$ & 0.50 & 0.20 & 0.46 \\
\hline
\end{tabular}

necessary step before comparing with experimental data since we have more control over parameters and physics involved. Future work should validate the model with experiments.

\subsection{Prediction for ITER}

We finally use the extended two-point model to predict the density and temperature at the inner and outer divertors for an ITER-like magnetic configuration. The list of relevant parameters are as follows: the major radius $R$ 


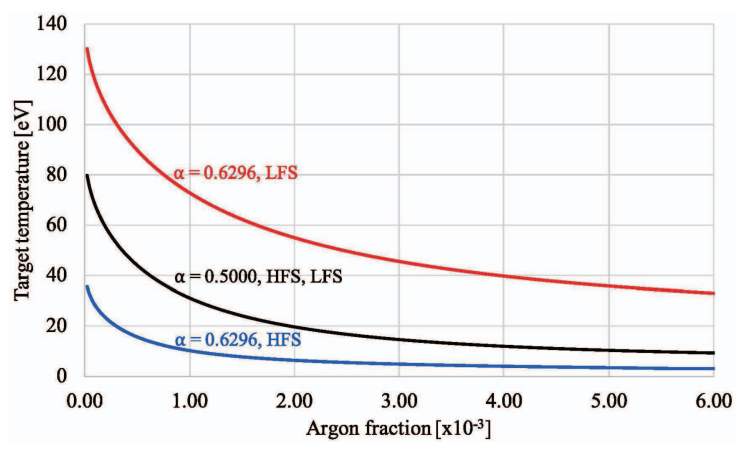

Fig. 4 The simulated target temperatures at the inner and outer divertors of ITER as a function of the argon fraction.

$=6.20 \mathrm{~m}$, the minor radius $a=2.00 \mathrm{~m}$, the plasma current $I_{P}=14.4 \mathrm{MA}$, the toroidal magnetic field $B_{T}=5.3 \mathrm{~T}$, the safety factor at the $95 \%$ of the poloidal flux $q_{95}=3.0$, the radiation power $P_{\text {rad }}=33 \mathrm{MW}$, the power crossing the separatrix $P_{\mathrm{u}}=92 \mathrm{MW}$, and the density at the upstream point $n_{u}=4.00 \times 10^{19} \mathrm{~m}^{-3}$.

It is further assumed that argon is the seeded impurity. Experimentally one observes that the asymmetric ratio between the power to the outer divertor and the power to the inner divertor can vary on a significant range, from 1:1 to 5:1 [23]. This ratio could be reduced if we allow neutral particles to commute between the two legs. As a representative average value, we use $P_{\mathrm{LFS}} / P_{\mathrm{HFS}}=1.7$, it corresponds to an asymmetry power parameter $\alpha=0.6296$. Figure 4 shows the predicted temperature at the inner and outer targets as a function of the argon fraction for $\alpha=0.6296$ and 0.50 . The corresponding values of the peak heat flux on both divertor targets are plotted in Fig. 5 . By construction, when $\alpha=0.5$, the temperatures on both divertor targets are the same. This is due to the symmetry of the particle and heat flux entering the SOL. Since the inner target has smaller plasma-wetted area, the peak heat flux on the inner divertor is higher than that on the outer divertor, see equation 21 . The differences of the temperatures and heat fluxes at both divertor targets become more pronounced when the power fraction $(\alpha)$ deviates from $\alpha=0.5$, see Figs. 4 and 5. In this case, both target temperature and peak heat flux on the outer divertor are higher than those on the inner divertor. The graphs show that the temperature and peak heat flux are decreasing as the argon fraction increases.

From Fig. 5, we also found that if the argon fraction is too low, the peak heat flux on the outer divertor may exceed the threshold value which is about $10 \mathrm{MW} / \mathrm{m}^{2}$ [18]. An impurity such as argon should be introduced in the region near the divertor. We found that the minimum concentration of argon required to keep the peak heat flux on the divertors below $10 \mathrm{MW}$ is about $4.85 \times 10^{-3}$ in agreement with more sophisticated simulations $[24,25]$.

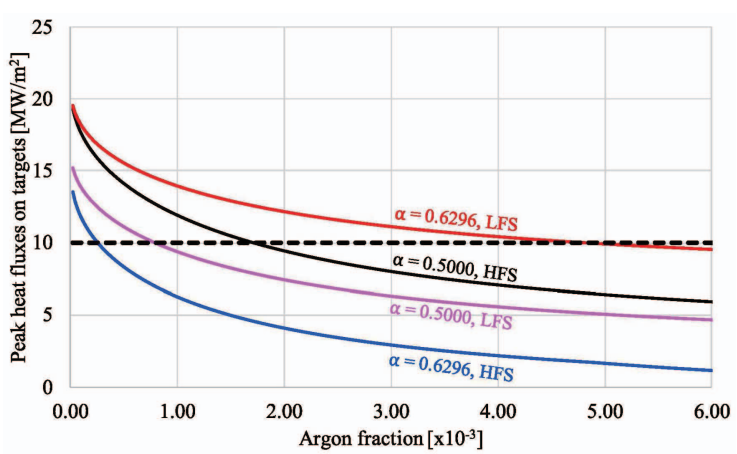

Fig. 5 The simulated peak heat fluxes at the inner and outer divertors of ITER as a function of the argon fraction. A dashed line denotes the threshold heat flux at the targets.

\section{Summary}

SYCOMORE is a system code that aims at modelling future fusion power plants with all subsystems included. It consists in a number of modules describing the different components of the reactor, that are all interconnected. For the computation of the density and temperature in the scrape-off layer, the SOLDIV code is used. However the previous version of SOLDIV assumed a symmetric SOL. In reality there is an imbalance of the heat flux between the two divertor legs, due to the ballooned transport, outward compression of the magnetic surfaces due to the Shafranov shift and asymmetry in the plasma wetted areas in the divertor. In this work, the SOLDIV module is improved to investigate this asymmetric transport in the SOL based on the extended two-point model. It is assumed that the outgoing fluxes of particles and heat from the plasma core enter the SOL at the upstream point whose location is determined as a fraction of the connection length $(\beta)$. The heat and particle fluxes then split into two parts: one flowing to the inner divertor, and the other towards the outer divertor. By introducing the imbalance factors of the energy flux $(\alpha)$ and connection length $(\beta)$, the transport equations become a set of nonlinear equations that can be numerically solved for the densities and temperatures at both divertor plates and the upstream point. We note that ELMs are not taken into account in this work. The two-point model is then validated with the transport code SolEdge2D-EIRENE for two WEST discharges. The densities and temperatures at the upstream and targets that are computed by the two codes agree within the average percentage difference of $18 \%$. Last but not least, the predictions of the densities, temperatures, and heat fluxes at both divertor targets of ITER are also reported. To keep the heat flux on the outer divertor below $10 \mathrm{MW} / \mathrm{m}^{2}$, the argon fraction should be about $4.85 \times 10^{-3}$ for an assumed density at the separatrix of $4 \times 10^{19} \mathrm{~m}^{-3}$.

\section{Acknowledgement}

This work was partially supported by the Thailand Research Fund under Contract No. MRG6180175, The- 
oretical and Computational Science Center (TaCS) at King Mongkut's University of Technology Thonburi, and Thailand Institute of Nuclear Technology. This is also part of a collaborative research project under the Center for Plasma and Nuclear Fusion Technology $(\mathrm{CPaF})$. This work was granted access to the HPC resources of Aix-Marseille University financed by the project Equip@Meso (ANR-10EQPX-29-01).

[1] P. Stangeby and G. McCracken, Nucl. Fusion 30, 1225 (1990).

[2] P.C. Stangeby et al., The plasma boundary of magnetic fusion devices, vol.224 (Institute of Physics Publishing Bristol, 2000).

[3] H. Bolt et al., J. Nucl. Mater. 307, 43 (2002).

[4] R. Pitts et al., J. Nucl. Mater. 438, S48 (2013).

[5] M. Roedig et al., Fusion Eng. Des. 61, 135 (2002).

[6] G. Federici et al., Fusion Eng. Des. 109, 1464 (2016).

[7] R. Wenninger et al., Nucl. Fusion 57, 016011 (2016).

[8] C. Reux et al., Nucl. Fusion 55, 073011 (2015).

[9] C. Reux et al., Fusion Eng. Des. (2018).
[10] A. Li-Puma, J.-C. Jaboulay and B. Martin, Fusion Eng. Des. 89, 1195 (2014).

[11] P. Ghendrih et al., J. Nucl. Mater. 438, S368 (2013).

[12] D. Galassi et al., Nucl. Fusion 57, 036029 (2017).

[13] A. Kallenbach et al., J. Nucl. Mater. 337, 381 (2005).

[14] J. Jean, Fusion Sci. Technol. 59, 308 (2011).

[15] W.H. Press, B.P. Flannery, S.A. Teukolsky, W.T. Vetterling et al., Numerical recipes, vol.2 (Cambridge university press Cambridge, 1989).

[16] D. Post, J. Abdallah, R. Clark and N. Putvinskaya, Phys. Plasmas 2, 2328 (1995).

[17] A. Mavrin, J. Fusion Energy 36, 161 (2017).

[18] D. Guilhem et al., J. Nucl. Mater. 196, 759 (1992).

[19] G. Telesca et al., Plasma Phys. Control. Fusion 53, 115002 (2011).

[20] L. Isoardi et al., J. Comput. Phys. 229, 2220 (2010).

[21] H. Bufferand et al., Nucl. Fusion 55, 053025 (2015).

[22] G. Ciraolo et al., Nucl. Mater. Energy 12, 187 (2017).

[23] C.S. Pitcher and P. Stangeby, Plasma Phys. Control. Fusion 39, 779 (1997).

[24] B. Braams, Contrib. Plasma Phys. 36, 276 (1996).

[25] D. Knoll, Nucl. Fusion 38, 133 (1998). 